\title{
Inadequação nutricional na dieta de atletas adolescentes
}

\section{Nutritional inadequacy in dietary intake of school athletes}

Jamille Mendonça Reinaldo¹, Danielle Góes da Silva², Renata Costa Matos',

Marinade Macedo Rodrigues Leite ${ }^{1}$, Raquel Simões Mendes-Netto ${ }^{2,3}$

${ }^{1}$ Curso de Nutrição, Universidade Federal de Sergipe (UFS) - São Cristóvão (SE), Brasil.

${ }^{2}$ Departamento de Nutrição, Centro de Ciências Biológicas e da Saúde, UFS - São Cristóvão (SE), Brasil.

${ }^{3}$ Programa de Pós-Graduação em Educação Física, UFS - São Cristóvão (SE), Brasil.

\section{RESUMO}

Introdução: Os atletas adolescentes têm uma demanda energética aumentada devido à prática competitiva de exercício físico, apresentando maiores riscos de inadequações nutricionais. Objetivo: Avaliar a ingestão alimentar de atletas escolares envolvidos em diferentes modalidades esportivas. Métodos: A amostra foi composta por 129 adolescentes, de ambos os gêneros, praticantes de tênis, natação, ginástica (artística e rítmica) e judô de equipes do município de Aracaju (SE), Brasil. Aplicou-se um questionário de frequência alimentar semiquantitativo, com auxílio de registro fotográfico, para estimar a ingestão de macro e micronutrientes e porções dos grupos alimentares. Resultados: Entre os analisados, 41,2\% eram do gênero feminino, com média de idade de 13,54 $\pm 2,45$ anos. Independente do gênero, os adolescentes mais velhos apresentaram maior ingestão energética e de macronutrientes $(p<0,05)$, porém com semelhança na prevalência de inadequação em relação aos mais novos. Com relação aos micronutrientes, a comparação entre os gêneros mostrou maior prevalência de inadequações entre meninas mais novas para vitamina E, cálcio e ferro. Entre os meninos, apenas a inadequação do cálcio foi significativamente maior para os mais novos. Independente do gênero e da idade, observou-se baixa ingestão de lácteos e hortaliças, além do elevado consumo de doces, carnes e ovos. Conclusão: A dieta dos atletas adolescentes estudados mostrou inadequação de macro e de micronutrientes, especialmente entre as atletas mais novas. Faz-se essencial o desenvolvimento de ações de aconselhamento nutricional individual ou em grupo com vistas a adquirir hábitos alimentares adequados.

Palavras-chave: adolescente; ingestão de alimentos; exercício.

\begin{abstract}
Introduction: Young athletes have an increased energy demand because of the competitive practice of physical exercise, presenting greater risks of nutritional inadequacies. Objective: To evaluate the dietary intake of school athletes who practice different sports by gender and age. Methods: The sample consisted of 129 adolescents of both genders, who were tennis, swimming, gymnastics (artistic and rhythmic), and judo athletes of sports teams in the city of Aracaju (SE), Brazil. A semiquantitative food frequency questionnaire was applied, which was also supported by a photographic record, to estimate the dietary intake of macro and micronutrients as well as food portions. Results: Among the young athletes analyzed, $41.2 \%$ were female with a mean age of $13.54 \pm 2.45$ years. Regardless of gender, older adolescents had higher energy and macronutrient intakes $(p<0.05)$, but presented similar prevalence of inadequacy in relation to the young. Regarding micronutrients, the comparison between genders showed a higher prevalence of inadequacy among younger girls for vitamin E, calcium and iron. Among boys, only the inadequacy of calcium was significantly higher among the younger athletes. Regardless of gender and age, there was a low intake of dairy products and vegetables and a high consumption of sweets, meats and eggs. Conclusion: The diet of adolescent athletes studied showed inadequacy of macro and micronutrients, especially among the younger athletes. It is essential to develop individual or in group nutritional counseling, so that they may acquire proper eating habits.
\end{abstract}

Keywords: adolescent; eating; exercise. 


\section{INTRODUÇÃO}

Durante a adolescência, fase entre 10 e 19 anos, o indivíduo passa por elevadas transformações biológicas, que são influenciadas por fatores ambientais, psicológicos, hereditários e nutricionais ${ }^{1}$. O crescimento e o desenvolvimento do adolescente são acompanhados também por mudanças comportamentais caracterizadas pela autonomia e poder de decisão de adoção ou não de novos hábitos. Com o avançar da idade, as escolhas pessoais do adolescente ganham prioridade sobre os hábitos alimentares adquiridos na família, e esses têm, progressivamente, mais controle do que comem, quando e onde 2 .

Nessa fase, observa-se uma rápida velocidade de crescimento, inferior somente ao primeiro ano de vida. Consequentemente, essas mudanças corporais exigem uma maior oferta energética. A nutrição inadequada entre os adolescentes pode interferir negativamente na maturação sexual e no crescimento saudável ${ }^{2}$.

A prática regular de exercício físico na adolescência é benéfica para a saúde física e intelectual, por contribuir no processo de crescimento, manutenção da massa magra, além de colaborar no desenvolvimento da saúde mental. $\mathrm{O}$ esporte não só previne patologias de elevada prevalência, como a obesidade e a hipertensão, mas também proporciona qualidade de vida ao indivíduo ${ }^{3}$. Entretanto, os adolescentes que praticam exercícios com o intuito competitivo possuem um gasto energético aumentado e, por isso, requerem maior demanda energética ${ }^{4}$.

Durante a atividade física intensa, é imprescindível que a ingestão energética seja suficiente para atingir as necessidades corporais, além da adequação de carboidratos, proteínas, água e eletrólitos, por afetar diretamente a composição corporal e o desempenho esportivo ${ }^{5}$. Em seu estudo, Couto et al. ${ }^{6}$ mostraram a relação entre a ingestão adequada de carboidrato e o desempenho esportivo em jovens corredores adolescentes, uma vez que os atletas com uma dieta de aproximadamente $7 \mathrm{~g} / \mathrm{kg} /$ dia nas 48 horas anteriores à atividade física concluíram uma prova de $10.000 \mathrm{~m}$ em menor tempo $(\mathrm{p}<0,05)$ que aqueles com consumo de $2,5 \mathrm{~g} / \mathrm{kg} / \mathrm{dia}$ de carboidrato.

Com o aumento das necessidades energéticas provocadas pelos exercícios, os adolescentes esportistas estão mais sujeitos às inadequações nutricionais - especialmente o gênero feminino - , o que resulta em maior risco de desenvolvimento de deficiências nutricionais, como a anemia e a osteoporose na vida adulta ${ }^{7}$.

Nas atletas em que é necessário o controle do peso corporal, como as ginastas e as patinadoras, o risco de deficiências nutricionais aumenta devido às práticas alimentares inadequadas, como as frequentes restrições calóricas que visam à melhora do rendimento profissional $^{8}$.

Por outro lado, o estudo de Daneshvar et al. ${ }^{9}$ mostrou que os jovens atletas lutadores do gênero masculino apresentaram ingestão calórica maior que as suas necessidades, o que contribuiu com a ingestão de vários nutrientes. Ao que parece, fatores como gênero, modalidade esportiva $^{7}$ e apoio familiar ${ }^{10}$ podem determinar diferenças na ingestão dietética diária do atleta adolescente.

Considerando que ainda são escassos os estudos que caracterizam a ingestão dietética de jovens atletas — principalmente no Brasil - e que os hábitos alimentares não se mostram semelhantes entre os gêneros e as diferentes idades da adolescência, o presente estudo teve por objetivo analisar, por gênero e idade, a alimentação de adolescentes atletas escolares envolvidos em diferentes modalidades esportivas.

\section{MÉTODOS}

Foram recrutados para o estudo 129 adolescentes (10 a 19 anos), de ambos os gêneros, praticantes de tênis, natação, ginástica (artística e rítmica) e judô, que residem na cidade de Aracaju (SE). Os adolescentes que compuseram a amostra faziam parte de equipes de destaque do estado, com participação em jogos de nível regional e nacional.

Os pais ou os responsáveis dos integrantes da pesquisa assinaram o Termo de Consentimento Livre e Esclarecido, conforme protocolo aprovado pelo Comitê de Ética em Pesquisa da Universidade Federal de Sergipe (CAAE - 0032.0.107.000-10). Foram excluídos os atletas que não frequentavam regularmente os treinos por estarem lesionados, além dos que apresentavam histórico de doenças metabólicas ou que faziam uso regular de medicamentos.

A coleta de dados foi realizada de 2009 a 2012. Os técnicos ajudaram na confecção de uma listagem com os nomes, gênero e local de treinamento dos adolescentes. Para obter os dados necessários dos atletas, foi aferido o peso corporal (Balança eletrônica Líder, escala de 100g) em horário anterior ao treinamento e, em seguida, foram aplicados dois questionários: o primeiro para a identificação pessoal, onde constam perguntas sobre histórico de doenças e uso de medicamentos, e o segundo - o Questionário de Frequência Alimentar (QFA) -, que avalia o consumo alimentar, além da ingestão hídrica habitual. As entrevistas aconteceram nos centros esportivos onde ocorriam os treinos e os questionários foram aplicados em horário anterior ou posterior ao treinamento. $\mathrm{O}$ estudo teve caráter transversal.

Para obter os dados dietéticos dos adolescentes foi aplicado o QFA, semiquantitativo referente aos últimos seis meses, adaptado de Slater et al. ${ }^{11}$. Para melhor reconhecimento das porções alimentares pré-estabelecidas, foi utilizado registro fotográfico de alimentos ${ }^{12-15}$.

O questionário possuía sete categorias de respostas que variavam de "nunca" a "duas ou mais vezes ao dia". Um padrão diário de ingestão foi obtido para cada alimento contido no QFA. Para isso, estabeleceu-se constantes para cada categoria de ingestão. A opção "nunca" não foi considerada como ingestão. Para as demais categorias estabeleceram-se constantes a partir da média dos dias da categoria (mês, semana, dia) dividida pelo número total de dias adaptado de Colluci ${ }^{16}$, ou seja, $1 /$ mês $=0,03(1 \div 30) ; 1$ a $3 /$ mês $=0,06[(1+3) \div 2] \div 30,1 /$ semana $=0,14$ $(1 \div 7) ; 2$ a $4 /$ semana $=0,43[(2+4) \div 2] \div 7,1 /$ dia $=$ valor obtido na medida padronizada; $2 /$ dia = dobro do valor obtido na porção padronizada.

A quantidade em gramas da porção padronizada do alimento foi multiplicada pela constante encontrada e, assim, obteve-se o consumo diário. Para quantificar a ingestão de energia e de nutrientes utilizou-se o software Virtual Nutri Plus, versão 2.0 Plus ${ }^{17}$. 
A classificação da ingestão de carboidratos e de proteínas em gramas por quilo de peso corporal $(\mathrm{g} / \mathrm{kg})$ foi realizada sobre a recomendação específica para atletas segundo literatura atual, sendo carboidratos de 5 a $7 \mathrm{~g} / \mathrm{kg}^{18}$ e proteínas de 1,3 a $1,8 \mathrm{~g} / \mathrm{kg}^{19}$. Já para os lipídeos, a classificação foi feita utilizando a recomendação que varia de 20 a $35 \%$ do valor energético total $\left(\right.$ VET) ${ }^{20}$. Os valores que não se encaixavam nesses intervalos foram considerados como inadequados. A avaliação de ingestão dietética de micronutrientes foi realizada com base nas Ingestões Dietéticas de Referência (Dietary References Intakes - DRI) ${ }^{21-24}$. Quando essa ingestão foi inferior à necessidade média estimada (Estimated Average Requirement - EAR) ${ }^{25}$, considerou-se indequação. Para a avaliação da ingestão hídrica, utilizou-se a recomendação de $30 \mathrm{~mL} / \mathrm{kg}$ por dia. Os valores inferiores foram classificados como inadequados ${ }^{26}$. Esses dados de ingestão, juntamente com as prevalências de inadequações, foram considerados de acordo com o gênero e faixa etária, segundo a indicação do Instituto de Medicina (IOM) ${ }^{27}$.

Para a obtenção do número de porções dos oito grupos alimentares consumidos pelos adolescentes, foram utilizados os dados de gramas por dia a partir do QFA. Ocorreu a separação dos alimentos em seus respectivos grupos e, em seguida, somou-se os valores de energia total. Esse valor energético foi dividido pelas calorias determinadas por Philippi et al..$^{28}$ para cada grupo alimentar. Assim, foi possível obter o número de porções consumidas por cada indivíduo. Em seguida, os dados foram apresentados em percentis (p25, p50 e p75), e analisada a frequência de inadequação de acordo com o número de porções recomendadas em cada grupo alimentar. Os dados foram armazenados no Excel e posteriormente analisados utilizando o programa SPSS, versão 19.0. A estatística descritiva compreendeu o cálculo da média, do desvio padrão e das frequências absoluta e relativa. A normalidade das variáveis foi verificada pelo teste Kolmogorov-Smirnov. Para a comparação das variáveis contínuas entre o gênero e a faixa etária, utilizou-se o teste Mann Whitney ou o Test $t$ de Student. Para a análise de associação, utilizou-se o teste de $\chi^{2}$ de Pearson. Adotou-se como significativos os valores de p inferiores a 0,05.

\section{RESULTADOS}

Os resultados fazem referência a 129 adolescentes praticantes de tênis $(n=22)$, natação $(n=41)$, ginástica artística e rítmica $(n=24)$

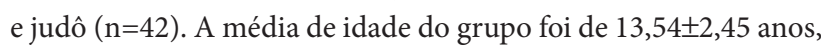
sendo a maioria do gênero masculino (58,8\%). O tênis foi a única modalidade formada somente por meninos.

Na Tabela 1 são apresentadas as médias dos valores de energia, de ingestão hídrica e de macronutrientes dos adolescentes de acordo com o gênero e a idade, bem como o percentual de indivíduos com consumo inadequado conforme as recomendações.

Ao comparar os gêneros na mesma faixa etária, observou-se que somente as variáveis fibras e lipídeos apresentaram diferenças significativas.

Tabela 1: Média e desvio padrão da ingestão hídrica, da energia e dos macronutrientes, e percentual de inadequação dos adolescentes de acordo com o gênero, em Aracaju, Sergipe, 2009 a 2012

\begin{tabular}{|c|c|c|c|c|c|}
\hline \multirow[b]{2}{*}{ Nutrientes } & \multirow[b]{2}{*}{ Recomendação } & \multicolumn{2}{|c|}{ Masculino $(n=74)$} & \multicolumn{2}{|c|}{ Feminino $(n=55)$} \\
\hline & & $\begin{array}{c}<14 \text { anos } \\
(n=39)\end{array}$ & $\begin{array}{c}\geq 14 \text { anos } \\
(n=35)\end{array}$ & $\begin{array}{c}<14 \text { anos } \\
(n=34)\end{array}$ & $\begin{array}{c}\geq 14 \text { anos } \\
(n=21)\end{array}$ \\
\hline Energia (kcal) & & $2.594,44 \pm 1.026,41$ & $3.548,00 \pm 1.178,37^{\star *}$ & $2.189,58 \pm 950,80$ & $2.914,21 \pm 907,90^{\circ}$ \\
\hline Ingestão Hídrica(mL) & $30 \mathrm{~mL} / \mathrm{kg}^{26}$ & $1.305,92 \pm 1134,40$ & $2.381,71 \pm 5.236,97$ & $1.475,97 \pm 1.023,60$ & $1.300 \pm 581,55$ \\
\hline$(\% \mathrm{I})$ & & $(66,7 \%)$ & $(77,1 \%)$ & $(47,1 \%)$ & $(61,9 \%)$ \\
\hline \multicolumn{6}{|l|}{ Carboidratos } \\
\hline Gramas totais & & $385,07 \pm 160,96$ & $515,78 \pm 176,27^{* *}$ & $330,03 \pm 158,80$ & $445,62 \pm 169,07^{b}$ \\
\hline Gramas/kg & $5-7 g / \mathrm{kg}^{18}$ & $8,65 \pm 4,56$ & $8,19 \pm 3,78$ & $8,27 \pm 5,05$ & $8,62 \pm 3,50$ \\
\hline$(\% \mathrm{I})$ & & $(76,3 \%)$ & $(82,9 \%)$ & $(80,0 \%)$ & $(61,9 \%)$ \\
\hline Fibra (g) & & $26,00 \pm 13,25$ & $33,47 \pm 14,81^{* * *}$ & $21,57 \pm 9,73$ & $25,17 \pm 9,34$ \\
\hline \multicolumn{6}{|l|}{ Lipídeos } \\
\hline Gramas totais & & $89,79 \pm 42,14^{*}$ & $120,90 \pm 56,66^{b}$ & $69,26 \pm 35,58$ & $94,02 \pm 34,13^{b}$ \\
\hline$\%$ do VET & 20 a $35 \%$ do VET $T^{\star \star \star}$ & $30,99 \pm 5,75$ & $30,03 \pm 6,60$ & $27,88 \pm 6,20$ & $28,67 \pm 3,99$ \\
\hline$(\% \mathrm{I})$ & & $(28,2 \%)$ & $(34,3 \%)$ & $(23,5 \%)$ & $(9,5 \%)$ \\
\hline \multicolumn{6}{|l|}{ Proteínas } \\
\hline Gramas totais & & $67,58 \pm 44,46$ & $114,40 \pm 77,14^{\star *}$ & $74,55 \pm 35,85$ & $99,79 \pm 36,20^{b}$ \\
\hline Gramas/kg & $1,2-1,8 \mathrm{~g} / \mathrm{kg}^{19}$ & $1,53 \pm 1,09$ & $1,56 \pm 1,07$ & $1,84 \pm 0,87$ & $1,82 \pm 0,69$ \\
\hline$(\% \mathrm{I})$ & & $(73,7 \%)$ & $(85,7 \%)$ & $(68,6 \%)$ & $(66,7 \%)$ \\
\hline
\end{tabular}

\% I: percentual de inadequação; \% do VET: Percentual do valor energético total; Burke et a/18.; Phillips e Vanloon ${ }^{19}$; Ryan ${ }^{26}$; *Diferença significativa (p $\left.\leq 0,05\right)$ entre gêneros na mesma faixa etária; ${ }^{*}$ Diferença significativa $(p \leq 0,05)$ entre faixas etárias do mesmo gênero; ***Ingestões Dietéticas de Referência - DRI, 2005. 
A ingestão de fibras foi maior entre os adolescentes do gênero masculino com idade igual ou superior a 14 anos e a de lipídeos (grama total) entre os menores de 14 anos, comparado às meninas das mesmas faixas etárias.

Quanto à análise dos dados dietéticos por gênero entre faixas etárias distintas, os esportistas com idade igual ou superior a 14 anos, tanto meninos quanto meninas, tiveram a ingestão significativamente maior de energia, carboidrato, lipídeo e proteína. Além desses nutrientes, os meninos com idade mais elevada possuíram o consumo de fibras superior ao dos meninos mais novos.

Em relação às recomendações de macronutrientes, identificou-se que mais de $55 \%$ dos adolescentes apresentaram

Tabela 2: Percentual de inadequação dos micronutrientes de acordo com o gênero e a faixa etária, em Aracaju, Sergipe, 2009 a 2012

\begin{tabular}{|c|c|c|c|c|}
\hline \multirow{3}{*}{ Nutrientes } & \multicolumn{4}{|c|}{ Percentual de Inadequação (\%) } \\
\hline & \multicolumn{2}{|c|}{ Masculino $(n=74)$} & \multicolumn{2}{|c|}{ Feminino $(n=55)$} \\
\hline & $\begin{array}{c}<14 \text { anos } \\
(n=39)\end{array}$ & $\begin{array}{c}\geq 14 \text { anos } \\
(n=35)\end{array}$ & $\begin{array}{c}<14 \text { anos } \\
(n=34)\end{array}$ & $\begin{array}{c}\geq 14 \text { anos } \\
(n=21)\end{array}$ \\
\hline Vittamina A (mg/d) & 10,5 & 17,1 & 28,6 & 14,3 \\
\hline Vitamina B1 (mg/d) & 0 & 0 & 5,7 & 0 \\
\hline Vitamina B2 (mg/d) & 2,6 & 8,6 & 20 & 9,5 \\
\hline Niacina (mg/d) & 5,3 & 8,6 & 17,1 & 0 \\
\hline Vitamina B6 (mg/d) & 5,3 & 5,7 & 11,4 & 4,8 \\
\hline Vitamina B12 (mg/d) & 23,7 & 42,9 & 40,0 & 28,6 \\
\hline Vitamina C (mg/d) & 5,3 & 8,6 & 2,9 & 4,8 \\
\hline Vitamina E (mg/d) & 7,9 & 11,4 & $28,6^{*}$ & 14,3 \\
\hline Cálcio (mg/d) & $76,3^{\star *}$ & 48,6 & $97,1^{* * *}$ & 76,2 \\
\hline Magnésio (mg/d) & 57,9 & 45,7 & 68,6 & 61,9 \\
\hline Ferro (mg/d) & 2,6 & 0 & $17,1^{* * *}$ & 0 \\
\hline Zinco (mg/d) & 50,0 & 37,1 & 62,9 & 33,3 \\
\hline
\end{tabular}

*Diferença significativa $(p \leq 0,05)$ entre gêneros na mesma faixa etária; **Diferença significativa $(p \leq 0,05)$ entre faixas etárias do mesmo gênero inadequações nutricionais, sendo representadas pela alta ingestão de carboidratos e de proteínas. Já para os lipídeos, o percentual foi reduzido, porém as inadequações também foram representadas pelo alto consumo. Para a ingestão hídrica, o percentual de inadequação variou entre 47,1 e 77,1\% (Tabela 1).

A prevalência de inadequação dos micronutrientes está disposta por gênero e faixa etária na Tabela 2. Entre os gêneros, notou-se nas meninas maior probabilidade de inadequação para o cálcio, o ferro e a vitamina E. Quanto à faixa etária, os mais novos são os mais afetados com a inadequada ingestão de nutrientes, especificamente de cálcio, em ambos os gêneros, e de ferro, entre as meninas.

Vale ressaltar que, além do cálcio, outros micronutrientes, como o magnésio, o zinco e a vitamina B12, tiveram uma baixa ingestão diária entre os esportistas estudados, porém não apresentaram diferenças significativas de inadequação entre os grupos (Tabela 2).

$\mathrm{Na}$ Tabela 3 é apresentada a análise da dieta dos adolescentes de acordo com o número de porções dos grupos alimentares. Diferente das tabelas anteriores, não houve um padrão diferencial entre gêneros e faixa etária, uma vez que o comportamento alimentar foi semelhante frente às inadequações, seja com relação ao consumo maior ou menor que as recomendações da pirâmide alimentar.

Os dados de prevalência de inadequação mostraram que os adolescentes apresentaram ingestão abaixo do recomendado para os grupos de frutas $(50,4 \%)$, hortaliças $(62,8 \%)$ e leite e derivados $(51,8 \%)$, enquanto o grupo de doces e carnes e ovos tiveram a ingestão acima das orientações para a população estudada, com valores de 79,1 e 69\%, respectivamente (Tabela 3).

\section{DISCUSSÃO}

Os adolescentes atletas não apresentaram diferenças expressivas $(\mathrm{p}<0,05)$ para os dados de ingestão dietética, quando comparados entre os gêneros. Todavia, ao confrontar os dados no mesmo gênero, observou-se que os adolescentes com idade igual ou maior que 14 anos possuíram maior ingestão calórica e de macronutrientes se

Tabela 3: Percentis $(25,50,75)$ do número de porções ingeridas e as análises de acordo com as recomendações da pirâmide alimentar entre os adolescentes do município de Aracaju, Sergipe, 2009 a 2011

\begin{tabular}{|c|c|c|c|c|c|c|}
\hline \multirow{2}{*}{ Grupos de alimentos } & \multirow{2}{*}{ REC $^{*}$} & \multicolumn{3}{|c|}{ Percentis } & \multirow{2}{*}{$\begin{array}{c}\text { Inferior à } \\
\text { recomendação } \\
(\%)\end{array}$} & \multirow{2}{*}{$\begin{array}{c}\text { Superior à } \\
\text { recomendação } \\
\text { (\%) }\end{array}$} \\
\hline & & p25 & p50 & p75 & & \\
\hline Cereais & $5-9$ & 4,62 & 6,34 & 8,35 & 30,2 & 20,2 \\
\hline Frutas & $3-5$ & 1,57 & 2,84 & 5,51 & 50,4 & 27,9 \\
\hline Hortaliças & $4-5$ & 0,87 & 1,60 & 3,29 & 79,1 & 12,4 \\
\hline Carnes e ovos & $1-2$ & 1,27 & 2,05 & 3,03 & 15,5 & 51,2 \\
\hline Leite e derivados & 3 & 0,93 & 1,92 & 3,47 & 69,0 & 20,9 \\
\hline Leguminosas & 1 & 0,67 & 1,55 & 2,41 & 35,7 & 37,2 \\
\hline Óleos e gorduras & $1-2$ & 0,71 & 1,41 & 2,31 & 38,0 & 23,3 \\
\hline Doces & $1-2$ & 1,78 & 3,15 & 4,67 & 3,9 & 62,8 \\
\hline
\end{tabular}

${ }^{*}$ REC: Recomendação - Philippi et al. ${ }^{28}$ 
comparados aos mais novos, tanto no grupo de meninos quanto no de meninas. No estudo, Martínez et al. ${ }^{29}$, ao analisar a ingestão de jovens nadadores semiprofissionais, verificaram diferenças entre gêneros para energia e proteína $(\mathrm{g} / \mathrm{kg})$.

A diferença de ingestão calórica e de nutrientes entre as faixas etárias pode ser explicada sob dois aspectos: (a) maior demanda energética dada pelas alterações corporais que acontecem na adolescência, como o estirão de crescimento e a formação dos caracteres primários e secundários $^{27}$ e (b) pela maior autonomia nas escolhas alimentares, que geralmente favorecem ao maior aporte calórico e que nem sempre é acompanhado de qualidade na alimentação ${ }^{30}$.

No presente estudo, a similaridade encontrada entre os gêneros na ingestão da maioria dos nutrientes possivelmente ocorreu por não existir diferença significativa $(\mathrm{p}<0,05)$ para os dados de valor calórico total da dieta, uma vez que a ingestão energética por parte do gênero feminino foi elevada quando comparada aos dados das atletas adolescentes avaliadas por Sousa et al. ${ }^{31}$, que foi na ordem de 2.032 a $2.401 \mathrm{kcal} /$ dia.

$\mathrm{Na}$ amostra estudada, a maioria das meninas $(63,6 \%)$ praticam esportes nos quais não há influência da massa corporal no rendimento esportivo. No judô, apesar da existência de divisões por categorias de peso em competições, os atletas geralmente reduzem a massa corporal em períodos próximos às competições e a recuperam em seguida, por utilizar métodos de perda rápida de peso ${ }^{32}$. No momento da análise, os atletas não estavam em períodos pré-competitivos, o que pode ter contribuído para a maior ingestão calórica das meninas.

Ao analisar o percentual de inadequação das proteínas e carboidratos, foi possível perceber que as alterações no consumo são marcadas pela elevada ingestão. $\mathrm{O}$ excesso de proteína contribui para o aumento da oxidação e, certamente, terá influência no rendimento se o aumento proteico for à custa de carboidrato e lipídeos, nutrientes também necessários para tolerar o nível de treinamento. Existem especulações sobre a elevação da proteína e do dano renal; entretanto, sabe-se que, em atletas saudáveis, não há evidências ${ }^{33}$.

A ingestão de proteína dos nadadores analisados por Martínez et al. ${ }^{29}$ foi também superior ao recomendado por $\mathrm{g} / \mathrm{kg} / \mathrm{dia}$. Acredita-se que, entre os atletas analisados, o elevado consumo de carnes e ovos provavelmente ocasionou alta ingestão proteica.

Apesar de o carboidrato atuar na manutenção dos níveis glicêmicos durante o exercício e na reposição do glicogênio muscular ${ }^{34}$, o consumo acima das recomendações gera estímulo a lipogênese, pela elevação da insulina e consequente alteração da composição corporal de atletas ${ }^{35}$.

Apesar dos esportistas com idade igual ou superior a 14 anos apresentarem maior ingestão calórica e de macronutrientes, ao se observar a Tabela 2, notou-se que micronutrientes importantes para o crescimento e o desenvolvimento dos adolescentes não seguiram o mesmo padrão, uma vez que possuíram altas prevalências de inadequação entre o cálcio, o magnésio, o zinco e a vitamina B12.

Mesmo apresentando elevado percentual de inadequação para cálcio, adolescentes atletas do presente estudo mostraram-se mais adequados nutricionalmente do que aqueles encontrados no estudo da Pesquisa de Orçamentos Familiares (POF), conduzido com adolescentes brasilei$\operatorname{ros}^{30}$. No entanto, no que tange à ingestão de cálcio, as atletas menores de 14 anos apresentaram prevalência de inadequação superior a 95\%, sendo o percentual semelhante ao do cenário nacional. Por outro lado, a prevalência de inadequação para zinco e vitamina B12 foi sensivelmente maior no presente estudo. Devem-se levar em conta, entretanto, os diferentes instrumentos utilizados nas duas pesquisas.

Juzwiak et al..$^{36}$, ao avaliarem o consumo alimentar de tenistas adolescentes do gênero masculino, verificaram que a ingestão foi menor que a recomendação para o cálcio e o magnésio nas duas faixas etárias ( $<14$ e $\geq 14$ anos). Com relação ao zinco, observou-se que as prevalências de inadequação para os indivíduos com idade igual ou maior que 14 anos foram similares ao presente estudo. Percebe-se que os adolescentes, sendo esportistas ou não, possuem uma alimentação similar no tocante às vitaminas e aos minerais, caracterizada pela baixa ingestão dos micronutrientes necessários para o crescimento e a maturação sexual.

Com o objetivo de comparar as prevalências de inadequações entre adolescentes, atletas ou não, de um mesmo grupo de estudantes escolares, Croll et al. ${ }^{37}$ mostraram que os adolescentes atletas apresentaram maior ingestão de nutrientes do que os não atletas, mesmo não atingindo a recomendação de vários micronutrientes. Os autores colocam que o envolvimento no esporte faz com que os adolescentes façam melhores escolhas, mesmo que ainda não sejam essencialmente adequadas do ponto de vista quantitativo.

A vitamina B12, como as outras do complexo B, é indispensável durante o estirão de crescimento. Suas principais fontes alimentares são os alimentos de origem animal ${ }^{34}$. Ao analisar a Tabela 3 , acreditase que o baixo consumo de lácteos possa ter contribuído para a inadequação dessa vitamina.

O inadequado consumo do magnésio, zinco e cálcio pode estar ligado à baixa ingestão de hortaliças e de leite e derivados, tendo em vista que eles são as principais fontes alimentares desses minerais e foram os grupos menos consumidos pelos adolescentes estudados (Tabela 3).

O magnésio atua como coadjuvante no processo de mineralização óssea, contribuindo para o pico de massa óssea. O zinco colabora para a função imunológica e antioxidante, sendo essencial no processo de maturação sexual ${ }^{15}$. O cálcio é imprescindível para o suprimento das demandas ósseas e prevenção de fraturas e osteoporose com o envelhecimento. Ele também está relacionado com a regulação da contração muscular, coagulação do sangue e condução nervosa ${ }^{15}$.

A osteoporose caracteriza-se pela diminuição da densidade mineral óssea e é um dos componentes da tríade da mulher atleta, juntamente com a menorreia e os distúrbios alimentares. Essa síndrome pode acometer atletas de nível competitivo ou não, sendo muitas vezes negada ou não diagnosticada. As atletas com maior risco são as praticantes de modalidades que exigem a manutenção de um baixo peso corporal, como as ginastas ${ }^{38}$.

A necessidade de ferro torna-se elevada durante períodos de rápido crescimento, como na adolescência, devido ao aumento da massa muscular, expansão do volume sanguíneo e capacidade respiratória ${ }^{15}$. Para as meninas esportistas, o requerimento de ferro é maior em função das perdas menstruais e da atividade física (hemólise por impacto $)^{39}$, o que pode explicar a diferença de inadequação entre gêneros. 
A ingestão adequada de ferro permite o transporte de oxigênio para o músculo, contribuindo assim com o desempenho esportivo. Todavia, a anemia por privação de ferro é provavelmente maior entre os atletas do que entre os indivíduos sedentários, especialmente entre as adolescentes do gênero feminino ${ }^{39}$, tendo em vista as necessidades aumentadas citadas anteriormente.

A inadequação de ferro entre a população estudada não foi tão elevada quanto aos outros micronutrientes já citados, possivelmente decorrente da elevada ingestão de carnes, ou seja, grupo alimentar com alto teor de ferro biodisponível.

A vitamina E é uma vitamina lipossolúvel, que possui como função a proteção contra os radicais livres. Suas principais fontes alimentares são os óleos de origem vegetal ${ }^{34}$. Supõe-se que a menor ingestão desse nutriente entre as meninas mais novas ocorreu devido à ingestão significativamente inferior de lipídeos em comparação com os outros grupos.

Ao confrontar os dados de ingestão dos grupos alimentares com o do estudo de Sousa e colaboradores ${ }^{31}$, que também compararam a ingestão de esportistas adolescentes, nota-se elevado consumo de doces e reduzido consumo de leites e derivados e hortaliças, dados esses semelhantes aos deste trabalho.

Percebe-se que as deficiências de nutrientes observadas sejam, possivelmente, decorrentes da baixa densidade nutricional dos alimentos preferencialmente consumidos pelos esportistas estudados. A correção da carência de vitaminas e minerais pode ocorrer com a maior inclusão de frutas, hortaliças, leites e derivados na alimentação habitual desses adolescentes.

O QFA utilizado não possui direcionamento a nenhum nutriente específico e teve a proposta de estratificar o consumo alimentar e de nutrientes dos adolescentes atletas analisados e, por consequência, pode limitar a interpretação dos resultados, podendo ser subestimado ou superestimado em alguns nutrientes. No entanto, o questionário utilizado foi validado em um estudo anterior ${ }^{11}$ para adolescentes, e devido à ausência de QFA para atletas adolescentes brasileiros, realizaram-se adaptações considerando aspectos como os hábitos alimentares do público-alvo e o consumo alimentar local na perspectiva de contemplar a alimentação da população estudada.

Em relação aos macronutrientes, o gênero masculino mostrou maior percentual de inadequação; quando analisada a inadequação nutricional de acordo com a faixa etária, independentemente do gênero, os adolescentes menores de 14 anos mostram-se mais inadequados nutricionalmente que aqueles com idade superior. A avaliação da adequação pela faixa etária entre os gêneros revelou que, no sexo feminino, a inadequação é maior nas adolescentes mais jovens, enquanto que, entre os homens, ocorre o oposto: o percentual de inadequação predomina nos jovens maiores de 14 anos.

Quanto aos micronutrientes, a inadequação é maior nas mulheres, quando comparados os gêneros. Entre essas adolescentes nota-se maior inadequação nas mais jovens; entretanto, no sexo masculino a faixa etária acima de 14 anos mostra uma maior inadequação nutricional. Independente do gênero, no quesito micronutrientes, os hábitos alimentares dos adolescentes mais jovens se mostraram piores do que os dos jovens mais velhos.

\section{AGRADECIMENTOS}

Aos atletas que aceitaram participar do projeto, aos técnicos pela contribuição e ao financiamento cedido pela concessão de bolsas do Programa de Iniciação Científica (PIBIC/CNPq e COPES/UFS).

\section{REFERÊNCIAS}

1. World Health Organization (WHO). Physical status: the use and interpretation of anthropometry. Geneva: WHO; 1995.

2. World Health Organization. Nutrition in adolescence: issues and challenges for the health sector - issues in adolescent health and development. Disponível em: http://whqlibdoc.who.int/ publications/2005/9241593660_eng.pdf. Acesso em: 19 jan. 2015.

3. Ganley T, Sherman C. Exercise and children's health: a little counseling can pay lasting dividends. Phys Sports Med. 2000;28(2):85-92

http://dx.doi.org/10.3810/psm.2000.02.699

4. Papadopoulou SK, Papadopoulou SD, Gallos GK. Macro- and micro-nutrient intake of adolescent Greek female volleyball players. Int J Sport Nutr Exerc Metabol. 2002;12(1):73-80.

5. American Dietetic Association; Dietitians of Canada; American College of Sports Medicine, Rodriguez NR, Di Marco NM, Langley S. American College of Sports Medicine position stand. Nutrition and athletic performance. Med Sci Sports Exerc. 2009;41(3):709-31.

http://dx.doi.org/10.1249/MSS.0b013e31890eb86
6. Couto PG, Bertuzzi R, de Souza CC, Lima HM, Kiss MA, de-Oliveira FR, et al. High Carbohydrate Diet Induces Faster Final Sprint and Overall 10,000-m Times of Young Runners. Pediatr Exerc Sci. 2015;27(3):355-63

http://dx.doi.org/10.1123/pes.2014-0211

7. Burke LM, Slater G, Broad EM, Haukka J, Modulon S, Hopkins WG. Eating patterns and meal frequency of elite Australian athletes. Int $J$ Sport Nutr Exerc Metab. 2003;13(4):521-38.

8. Dwyer J, Eisenberg A, Prelack K, Song WO, Sonneville K, Ziegler P. Eating attitudes and food intakes of elite adolescent female figure skaters: a cross sectional study. J Int Soc Sports Nutr. 2012;9(1):53. http://dx.doi.org/10.1186/1550-2783-9-53

9. Daneshvar P, Hariri M, Ghiasvand R, Askari G, Darvishi L, Iraj B et al. Dietary behaviors and nutritional assessment of young male isfahani wrestlers. Int J Prev Med. 2013;4(Suppl 1):S48-52.

10. Iglesias-Gutiérrez E, García-Rovés PM, García A, Patterson AM. Food preferences do not influence adolescent high-level athletes' dietary intake. Appetite. 2008;50(2-3):536-43. http://dx.doi.org/10.1016/j.appet.2007.11.003 
11. Slater B, Philippi ST, Marchioni DM, Fisberg RM. Validação de Questionário de Frequência Alimentar - QFA: considerações metodológicas. Rev Bras Epidemiol. 2003;6(3):200-8. http://dx.doi.org/10.1590/S1415-790X2003000300003

12. Zabotto CB, Vianna RPT, Gil MF. Registro fotográfico para inquéritos dietéticos: utensílios e porções. Goiânia: UFG; 1996.

13. Sales RL, Costa NMB, Silva MMS. Avaliando o consumo alimentar por fotos. [CD-ROM]. Viçosa: UFV; 2004

14. Lopez RPS, Botelho RBA. Álbum fotográfico de porções alimentares. São Paulo: Metha; 2008.

15. Vitolo MR. Nutrição: da gestação ao envelhecimento. Rio de Janeiro: Rubio; 2008.

16. Colucci ACA, Philippi ST, Slater B. Desenvolvimento de um questionário de frequência alimentar para avaliação do consumo alimentar de crianças de 2 a 5 anos de idade. Rev Bras Epidemiol. 2004; 7(4):393-401

http://dx.doi.org/10.1590/S1415-790X2004000400003

17. O Software Virtual Nutri Plus. Virtual NutriPlus. Versão 2.0. Disponível em: https://linux.ime.usp.br/ cef/mac499-04/monografias/gewerton/ Software.htm. Acesso em: 21 jan. 2016.

18. Burke LM, Hawley JA, Wong SH, Jeukendrup AE. Carbohydrates for training and competition. J Sports Sci. 2011;29(Suppl 1):S17-27

http://dx.doi.org/10.1080/02640414.2011.585473

19. Phillips SM, Van Loon LJ. Dietary protein for athletes: from requirements to optimum adaptation. J Sports Sci. 2011;29(Suppl 1):S29-38.

http://dx.doi.org/10.1080/02640414.2011.619204

20. Institute of Medicine of the National Academies. Dietary reference intakes for energy, carbohydrate, fiber, fat, fatty acids, cholesterol, protein and amino acids (macronutrients). Washington: The National Academies Press; 2005. Disponível em: http://www.nap. edu/read/10490/chapter/1. Acesso em: 12 maio 2016.

21. Institute of Medicine. Dietary reference intakes for thiamin, riboflavin, niacin, vitamin B6, folate, vitamin B12, pantothenic acid, biotin, and choline. Washington: National Academies Press; 1998.

22. Institute of Medicine. Dietary reference intakes for vitamin C, vitamin E, selenium, and carotenoids. Washington: National Academy Press; 2000.

23. Institute of Medicine. Dietary reference intakes for vitamin A, vitamin $\mathrm{K}$, arsenic, boron, chromium, copper, iodine, iron, manganese, molybdenum, nickel, silicon, vanadium, and zinc. Washington: National Academy Press; 2001

24. Institute of Medicine. Dietary Reference Intakes for Calcium and Vitamin D. Washington: The National Academies Press, 2011

25. National Research Council. Recommended dietary allowances. 10 ed. Washington: National Academy Press; 1989.

26. Ryan M. Complete guide to sports nutrition. Boulder: Velopress; 1999
27. Institute of Medicine. Food and Nutrition Board. Standing Committee on the Scientific Evaluation of Dietary Reference Intakes. Dietary reference intakes: applications in dietary assessment. Washington: National Academy Press; 2000.

28. Philippi ST, Latterza AR, Cruz ATR, Ribeiro LC. Pirâmide Alimentar Adaptada: guiapara escolha dos alimentos. Rev Nutr. 1999;12(1):65-80

29. Martínez S, Pasquarelli BN, Romaguera D, Arasa C, Tauler P, Aguiló A. Anthropometric characteristics and nutritional profile of young amateur swimmers. J Strength Cond Res. 2011;25(4):1126-33.

http://dx.doi.org/10.1519/JSC.0b013e3181d4d3df

30. Veiga GV, Da Costa RS, Araújo MC, Souza AM, Bezerra IN Barbosa FS, et al. Inadequação do consumo de nutrientes entre adolescentes brasileiros. Rev Saúde Pública. 2013;47(Supp 1):S212-21.

http://dx.doi.org/10.1590/S0034-89102013000700007

31. Sousa EF, Da Costa TH, Nogueira JA, Vivaldi LJ. Assessment of nutrient and water intake among adolescents from sports federations in the Federal District, Brazil. Br J Nutr. 2008;99(6): 1275-83.

http://dx.doi.org/10.1017/S0007114507864841

32. Artioli GG, Gualano B, Franchini E, Scagliusi FB, Takesian M, Fuchs $\mathrm{M}$, et al. Prevalence, magnitude, and methods of rapid weight loss among judo competitors. Med Sci Sports Exerc. 2010;42(3):436-42. http://dx.doi.org/10.1249/MSS.0b013e3181ba8055

33. Tipton KD, Wolfe RR. Protein and amino acids for athletes. Sports Sci. 2004;22(1):65-79 http://dx.doi.org/10.1080/0264041031000140554

34. Gallagher ML. Os Nutrientes e seu metabolismo In: Mahan LK, Escott-Strump S. Krause: alimentos, nutrição e dietoterapia. 12 ed. Rio de Janeiro: Elsevier; 2010. p.39-143.

35. Kersten S. Mechanisms of nutritional and hormonal regulation of lipogenesis. EMBO Rep. 2001;2(4):282-6. http://dx.doi.org/10.1093/embo-reports/kve071

36. Juzwiak CR, Amancio OMS, Vitalle MSS, Pinheiro MM, Szejnfeld VL. Body composition and nutritional profile of male adolescent tennis players. J Sports Sci. 2008;26(11):1209-17. http://dx.doi.org/10.1080/02640410801930192

37. Croll JK, Neumark-Sztainer D, Story M, Wall M, Perry C, Harnack $\mathrm{L}$. Adolescents involved in weight-related and power team sports have better eating patterns and nutrient intakes than non-sportinvolved adolescents. J Am Diet Assoc. 2006;106(5):709-17. http://dx.doi.org/10.1016/j.jada.2006.02.010

38. Leitão MB, Lazzoli JK, Oliveira MAB, Nóbrega ACL, Silveira GG, Carvalho T, et al. Posicionamento oficial da Sociedade Brasileira de Medicina do Esporte: atividade física e saúde na mulher. Rev Bras Med Esporte. 2000;6(6):215-20. http://dx.doi.org/10.1590/S1517-86922000000600001

39. Beard J, Tobin B. Iron Status and exercise. Am J Clin Nutr 2000:72(Suppl 2):594S-7S 